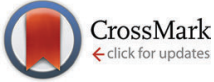

Cite this: Phys. Chem. Chem. Phys., 2016, 18, 3575

Received 29th October 2015 Accepted 21st December 2015 DOI: $10.1039 / \mathrm{c} 5$ cp06584d

www.rsc.org/pccp

\section{Phosphorescence quenching of fac-tris(2-phenylpyridyl)iridium(III) complexes in thin films on dielectric surfaces}

\author{
J. C. Ribierre, ${ }^{a}$ A. Ruseckas, ${ }^{a}$ S. V. Staton, ${ }^{b}$ K. Knights, ${ }^{b}$ N. Cumpstey, ${ }^{b}$ P. L. Burn* ${ }^{\text {bc }}$ \\ and I. D. W. Samuel*a
}

We study the influence of the film thickness on the time-resolved phosphorescence and the luminescence quantum yield of fac-tris(2-phenylpyridyl)iridium(III) [Ir(ppy) 3 -cored dendrimers deposited on dielectric substrates. A correlation is observed between the surface quenching velocity and the quenching rate by intermolecular interactions in the bulk film, which suggests that both processes are controlled by dipole-dipole interactions between $\operatorname{Ir}(\mathrm{ppy})_{3}$ complexes at the core of the dendrimers. It is also found that the surface quenching velocity decreases as the refractive index of the substrate is increased. This can be explained by partial screening of dipole-dipole interactions by the dielectric environment.

\section{Introduction}

Iridium(III) complexes have attracted intensive attention as phosphorescent emitters for organic light-emitting diodes (OLEDs) because they can have internal quantum efficiencies of $100 \%{ }^{1-5}$ Their short triplet excited state lifetime $(\sim \mu \mathrm{s})$ enables high brightness devices and their good color tunability make them particularly attractive for displays. Recently, electrophosphorescent devices based on an exciplex-forming co-host doped with an iridium(III) complex emitter have been reported with external quantum efficiencies exceeding 35\%. ${ }^{6}$ Despite the recent progress in the development of high performance phosphorescent and thermally-assisted delayed fluorescence (TADF) OLEDs, ${ }^{6-8}$ their efficiency is often reduced due to triplet quenching by intermolecular interactions, exciton-exciton annihilation and exciton-polaron interactions, ${ }^{9-12}$ which can be minimised by device and materials engineering. For instance, triplet-triplet annihilation and concentration quenching losses can be reduced in phosphorescent organic thin films using iridium(III) complexes within a dendritic structure. Surface quenching of phosphorescence has also been observed in very thin films deposited on

\footnotetext{
${ }^{a}$ Organic Semiconductor Centre, SUPA, School of Physics and Astronomy, University of St Andrews, North Haugh, St Andrews, Fife KY16 9SS, UK. E-mail: idws@st-andrews.ac.uk

${ }^{b}$ Department of Chemistry, University of Oxford, Chemistry Research Laboratory, Mansfield Road, OX1 3TA Oxford, UK

${ }^{c}$ Centre for Organic Photonics and Electronics, School of Chemistry and Molecular Biosciences, University of Queensland, Queensland 4072, Australia.

E-mail:p.burn2@uq.edu.au
}

fused silica substrates, but the mechanism by which this occurs has still not been elucidated. ${ }^{12,13}$ The recent advances in organic electroluminescent materials and devices make it essential to fully understand all these quenching mechanisms.

In this paper, we explore surface quenching of phosphorescence in films of several fac-tris(2-phenylpyridyl)iridium(III) $\left[\operatorname{Ir}(\mathrm{ppy})_{3}\right]$-cored dendrimers with different spacing between them, with the spacing controlled by the structure of the dendrimer as well as by blending with a large optical gap host. ${ }^{14-19}$ The molecular structures of the first generation $\left[\operatorname{Ir}(\mathrm{ppy})_{3}\right]$-cored dendrimers used in this work are shown in Fig. 1. We report a study on the thickness dependence of the decay of the dendrimer thin film phosphorescence and describe the results using a model based on surface quenching of triplets at the top and bottom interfaces of the film. The roles of exciton diffusion, the bulk photophysical properties of the dendrimer films and the refractive index of the substrates on the surface quenching effects are discussed, providing important insights into the triplet quenching mechanisms in phosphorescent organic thin films. Our results not only broaden our understanding of triplet-state photophysics, but are also of practical interest for the development of light-emitting devices, in particular electrophosphorescent organic field-effect transistors. ${ }^{20}$

\section{Experimental section}

Dendrimer films were prepared in the ambient atmosphere of a cleanroom by spin-coating a chloroform solution onto precleaned substrates. The substrates were composed of fused 

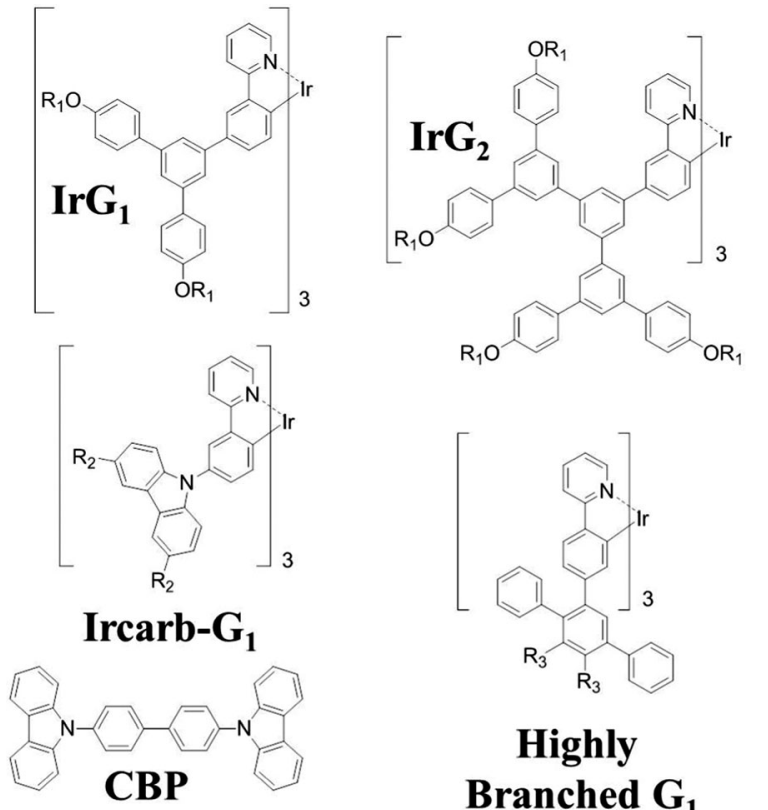

Fig. 1 Chemical structures of the macromolecules used in this work $\mathrm{R}_{1}=2$-ethylhexyl, $\mathrm{R}_{2}=\left(9,9\right.$-di- $n$-propylfluoren-2-yl) and $\mathrm{R}_{3}=4$ - $(9,9$-di$n$-propylfluoren-2-yl)phenyl.

silica, borosilicate glass, N-SK10 and SF15 glasses, sapphire, indium tin oxide (ITO) or a poly-(ethylenedioxythiophene):poly(styrenesulfonicacid) (PEDOT:PSS) (Baytron P, Bayer AG, Germany) layer deposited by spin-coating onto a fused silica substrate. The PEDOT:PSS film was then baked at $110{ }^{\circ} \mathrm{C}$ for 10 minutes before deposition of the dendrimer films. In addition, a $200 \mathrm{~nm}$ poly( $N$-vinylcarbazole) (PVK) (Sigma-Aldrich, $M_{\mathrm{w}}=1100000$ ) layer was spin-coated onto a fused silica substrate from a chlorobenzene solution and then dendrimer films were deposited from toluene. It has been previously shown that toluene does not dissolve PVK and enables the fabrication of a clean and sharp heterojunction interface, and we assume this is the case in this work. ${ }^{21}$ The concentration of the dendrimer and 4, $4^{\prime}$-bis( $N$-carbazolyl)-1, $1^{\prime}$ biphenyl (CBP) blend solutions varied typically from $1 \mathrm{mg} \mathrm{ml}^{-1}$ to $25 \mathrm{mg} \mathrm{ml}^{-1}$ in order to obtain film thicknesses ranging from 5 to $200 \mathrm{~nm}$. Film thicknesses were measured by variable angle spectroscopic ellipsometry (J.A. Woolam Co., Inc. M-2000DI system) and from the absorbance measured using a Cary Varian model 300 absorption spectrophotometer. The refractive indices of the substrates and the dendrimer films were also determined from ellipsometry. The photoluminescence quantum yield (PLQY) of each film was determined using an integrating sphere purged with flowing nitrogen. ${ }^{22}$ The samples were excited by a helium-cadmium laser with a wavelength of $325 \mathrm{~nm}$ or by a gallium nitride laser diode emitting at $407 \mathrm{~nm}$ to avoid absorption by some of the substrates. Time-resolved PL measurements were carried out using the time-correlated single-photon counting technique. The samples were excited at $390 \mathrm{~nm}$ by a pulsed laser diode (Picoquant, model PLS 370) giving 10 pJ per pulse at a repetition rate of $20 \mathrm{kHz}$ and were kept in a vacuum of $<8 \times 10^{-4}$ mbar.

\section{Results}

\subsection{Thickness dependence of the phosphorescence}

Fig. 2 shows the phosphorescence decays measured for neat dendrimer films of different thicknesses. Note that these decays were measured at low excitation densities (lower than $10^{17} \mathrm{~cm}^{-3}$ ) in order to avoid quenching of the emission by triplet-triplet exciton annihilation. ${ }^{12}$ It can be seen that the decays become faster in thin films. Strong thickness dependence of the decay time is observed in IrG1, Ircarb-G1 and the highly branched G1-dendrimer. The $80 \mathrm{wt} \%$ IrG1: CBP blend showed a weaker dependence, whereas the decay times for films of IrG2 and the $20 \mathrm{wt} \%$ IrG1: CBP blend was independent of thickness. Note that for these two latter materials, the identical decays in Fig. 2 had to be offset vertically to distinguish between them. A potential explanation for the thickness dependence of the decay time for the neat G1-dendrimer films could be that the photonic mode density at the dielectric interface could affect the radiative emission process. ${ }^{23-26}$ However, we can rule this out because if it was the changes in radiative lifetime due to microcavity effects would be similar for all materials and the dependence of the phosphorescence decays would not vary between materials.

In order to study the dependence of the radiative $k_{\mathrm{R}}$ and the non-radiative $k_{\mathrm{NR}}$ deactivation rates on film thickness using standard equations: PLQY $=k_{\mathrm{R}} /\left(k_{\mathrm{R}}+k_{\mathrm{NR}}\right)$ and $1 / \tau=k_{\mathrm{R}}+$ $k_{\mathrm{NR}}$, we have measured the PLQY of the neat IrG1 films deposited on fused silica substrates. This dendrimer was chosen for these measurements because its basic photophysical properties have been intensively studied in the past and are well documented. ${ }^{12,14,15,19}$ An excitation wavelength of $325 \mathrm{~nm}$ was used in this case to get sufficient absorbance. The PLQY of the $180 \mathrm{~nm}$ thick film was found to be $68 \pm 7 \%$, dropping to $54 \pm 5 \%$ and $29 \pm 3 \%$ for film thicknesses of 140 and $60 \mathrm{~nm}$ respectively. Due to the slightly non-single exponential character of some of the PL decays, we assumed single light-emitting species and we used the time interval during which the PL intensity decayed to $1 / e$ of the initial intensity to estimate the PL lifetime $\tau$. The results give a slight decrease of $k_{\mathrm{R}}$ values from
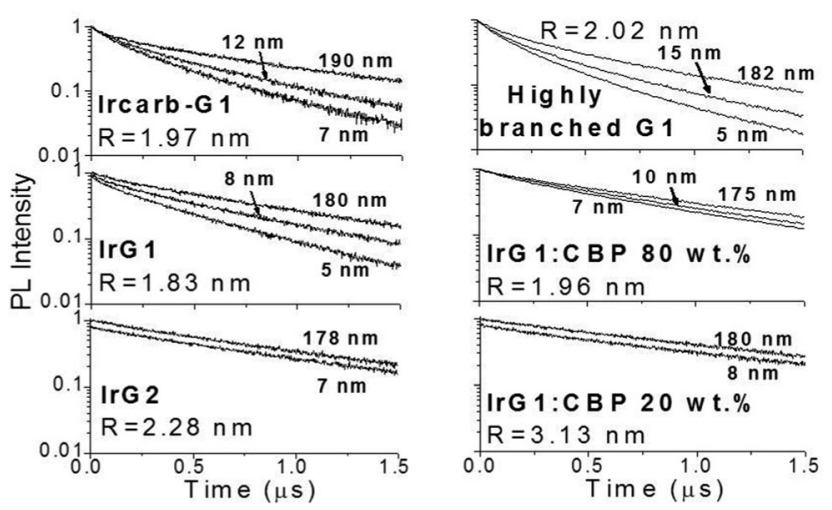

Fig. 2 Phosphorescence decay of dendrimer films with different thickness on fused silica substrates. The decays for IrG1: CBP (20:80 wt\%) and IrG2 films are independent of film thickness and have been shifted vertically for clarity. The average intermolecular spacing $R$ between the iridium(III) complex cores is reported in the insets. 
$7.5 \pm 0.7 \times 10^{5} \mathrm{~s}^{-1}$ for the $180 \mathrm{~nm}$ thick film to $5 \pm 0.4 \times$ $10^{5} \mathrm{~s}^{-1}$ for the $60 \mathrm{~nm}$ thick film and a three-fold increase of $k_{\mathrm{NR}}$ from $4 \pm 0.4 \times 10^{5} \mathrm{~s}^{-1}$ for the $180 \mathrm{~nm}$ film to $12 \pm 1 \times 10^{5} \mathrm{~s}^{-1}$ for the $60 \mathrm{~nm}$ film. This indicates that the decrease of the phosphorescence decay time in thin films is mainly due to an increase in the non-radiative decay rate. The thickness dependence of the PLQY has important ramifications for analysis of the different parameters involved in the expression of OLED external quantum efficiencies such as the light out-coupling efficiency or the singlet-triplet factor.

\subsection{Surface quenching of the phosphorescence}

The faster decay observed for the thinner films could be explained by quenching at one of the film surfaces, either the film/substrate or the film/vacuum interface. The dynamics of the exciton density $N(x, t)$ at the distance $x$ from the quenching surface can be described using the diffusion equation:

$$
\frac{\partial N(x, t)}{\partial t}=-k N(x, t)+D \frac{\partial^{2} N(x, t)}{\partial x^{2}}
$$

where $k$ is the exciton decay rate in the bulk and $D$ is the exciton diffusion constant. We define the boundary condition at the quenching interface as $D \partial N(x, t) / \partial x=v N(x, t)$ at $x=0$, where $v$ is the surface quenching velocity. At the non-quenching interface $\partial N(x, t)=0$ at $x=d$, where $d$ is the film thickness. In the limiting case of fast exciton diffusion, which corresponds to $D \gg v d$, the gradient of the exciton density is independent of $x$ and the average exciton density in the film $N_{\mathrm{av}}(t)=\frac{1}{d} \int_{0}^{d} N(x, t) \mathrm{d} x$, which is proportional to the measured time-resolved phosphorescence intensity can be described, assuming that the film is optically thin so that the initial excitations are uniform in the film, by a simple rate equation:

$$
\frac{d N_{\mathrm{av}}}{\mathrm{d} t}=-k N_{\mathrm{av}}-\frac{v N_{\mathrm{av}}}{d}
$$

In previous work, we have demonstrated that the assumption of fast exciton diffusion is applicable in neat films of iridium(III) complex-cored dendrimers thinner than $20 \mathrm{~nm} .{ }^{12}$ Exciton density in the bulk of the film, noted $N_{\mathrm{b}}$, can be described using a rate equation $\frac{\mathrm{d} N_{\mathrm{b}}}{\mathrm{d} t}=-k N_{\mathrm{b}}$. Introducing a new function $g(t)=\frac{N_{\mathrm{av}}}{N_{\mathrm{b}}}$ and solving eqn (2) we get:

$$
g(t)=\exp (-v t / d)
$$

where $g(t)$ can be obtained by taking a ratio of the phosphorescence intensity measured in thin films to that in a thick film. It is worth noting that the thickness dependence of the PL decays is found to be small for phosphorescent dendrimer films thicker than $50 \mathrm{~nm}$ and we thus assume that our thickest films are thick enough for the proposed method. In addition, it should be noted that our analysis is based on assumption of uniform excitation density and that we do not need to take into account the absorption profile in the thick film when the films are excited from the vacuum side, where surface quenching is not observed, as will be shown below. In addition, it should be
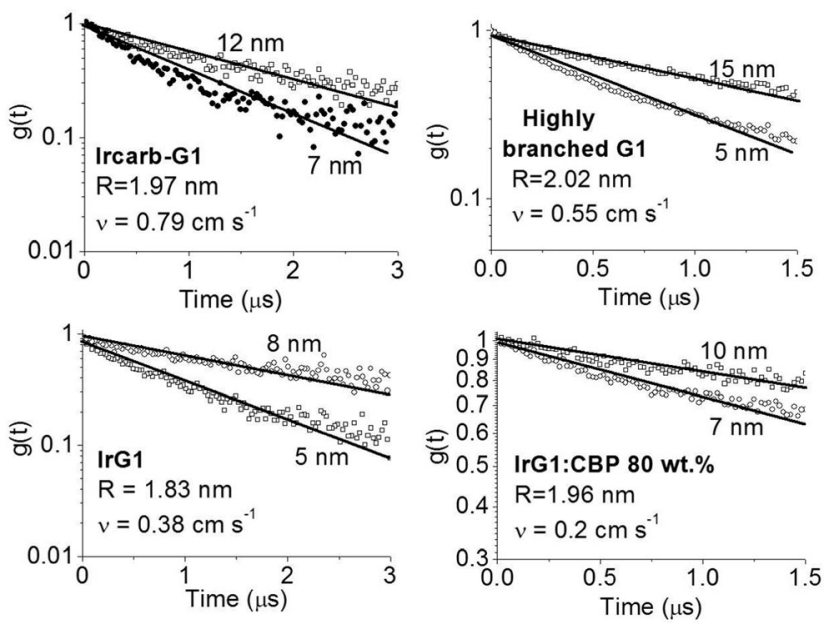

Fig. 3 Ratio of the phosphorescence intensity $g(t)$ measured for thin films of given thicknesses on fused silica substrates to that of the limiting case of a thick film. Solid lines are fits to an exponential decay and the values of the surface quenching velocities $v$ obtained using eqn (3) are given for each material as insets. $R$ is the average intermolecular spacing between the iridium(III) complex cores.

noted that even when the samples are photoexcited through the substrate, the absorption of the light at the excitation wavelength in the $15 \mathrm{~nm}$ layer near the substrate is $<0.05$, and hence only about $8 \%$ of excitons are affected by surface quenching in the $180 \mathrm{~nm}$ thick films. The results in Fig. 3 show that $g(t)$ for different film thicknesses can be fitted well by eqn (3) with a single value of $v$ for each material, which is given in the inset. In case of the IrG2 and IrG1 : CBP (20:80 wt\%) films no surface quenching was observed, and thus $v=0$.

To obtain a better understanding of the surface quenching we have investigated how it correlates with the triplet exciton diffusion and the non-radiative decay rate in the bulk of the materials. From the triplet-triplet exciton annihilation data reported elsewhere, ${ }^{12}$ we calculated the exciton diffusion constant $D$ using $\gamma=4 \pi D R_{\text {a }}$, where $\gamma$ is the annihilation constant, $R_{\mathrm{a}}$ is the annihilation radius at which annihilation is faster than hopping and is taken to be the spacing distance $R$ between two neighbouring iridium(III) complex cores. The intermolecular spacing $R$ was calculated by assuming the dendrimers are hard spheres and the films have a density of $1.1 \mathrm{~g} \mathrm{~cm}^{-3} \cdot{ }^{27}$ Fig. 4 shows the $D$ values decreasing with an increase of $R$, which confirms that exciton diffusion can be controlled by the attachment of different types and generations of dendrons, or by blending the dendrimers in a host at various concentrations. $^{12,28,29}$ The surface quenching velocity $v$ also decreases with an increase in $R$ for $R>1.97 \mathrm{~nm}$. However, two materials with the shortest intermolecular spacing $R$ and the highest triplet diffusivity show lower values of $v$ than the films of IrCarbG1 and of highly branched G1 with $R \sim 2 \mathrm{~nm}$. This indicates that exciton diffusion is not always a first order parameter responsible for phosphorescence quenching at an interface.

In order to estimate the phosphorescence quenching rate in the film bulk, we measured the PLQY values in $180 \mathrm{~nm}$ thick 


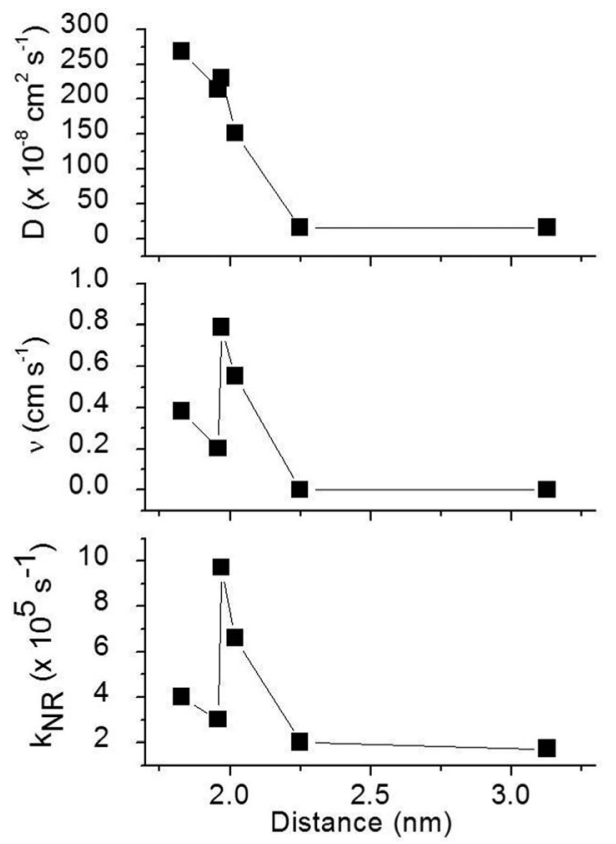

Fig. 4 Triplet exciton diffusion coefficient $D$, surface quenching velocity $v$ of the thin films, and the non-radiative decay rate $k_{N R}$ in the bulk films as a function of the intermolecular distance between complexes $R$.

films where surface quenching is negligible. These values are found to be in good agreement with the ones previously reported. ${ }^{12}$ The non-radiative decay rate $k_{\mathrm{NR}}$ was calculated using the phosphorescence lifetime determined as the time interval during which the PL intensity decayed to $1 / e$ of the initial intensity. The $1 / e$ decay time accounts for emission from a strongly emitting state which gives a dominant contribution to the photoluminescence quantum yield. We did not use an average phosphorescence lifetime because of a possible contribution of aggregates to weak long-lived phosphorescence. As all the films studied here show aggregation-induced quenching except $20 \mathrm{wt} \% \mathrm{IrG} 1$ : CBP and the IrG2, it is possible that dispersed and aggregated molecules both contribute to phosphorescence with different radiative rates. A previous study reported that the non-radiative decay rate for these types of phosphorescent dendrimers was a combination of intramolecular and intermolecular quenching processes. ${ }^{12}$ It was found that $k_{\mathrm{NR}}$ converged to a value $1.8 \times 10^{5} \mathrm{~s}^{-1}$ in the low concentration IrG1: CBP blend regime, with this value attributed to intramolecular non-radiative deactivation, assuming an even distribution of the dendrimer in the host. ${ }^{12}$ Fig. 4 shows that $k_{\mathrm{NR}}$ and the surface quenching velocity $v$ both decrease sharply when the intermolecular spacing $R$ increases above $2 \mathrm{~nm}$ and also shows smaller values for the two materials with $R<2 \mathrm{~nm}\left(0.17\right.$ and $\left.0.38 \mathrm{~cm} \mathrm{~s}^{-1}\right)$. In fact, $v$ shows a roughly linear dependence on $k_{\mathrm{NR}}$ and intersects the $x$-axis at $k_{\mathrm{NR}} \approx$ $2 \times 10^{5} \mathrm{~s}^{-1}$ (Fig. 5), which is close to the intramolecular nonradiative decay rate. It is worth noting that phosphorescence quenching at the surface is negligible in the same two materials which show no aggregation-induced quenching in the bulk of the film. Likewise, the strongest surface quenching effect is

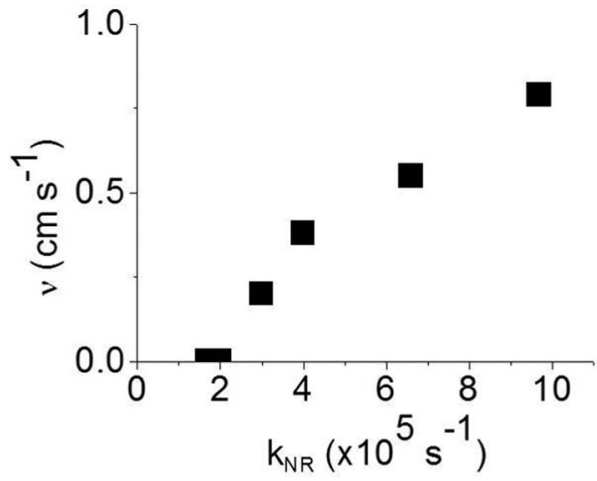

Fig. 5 Surface quenching velocity $v$ in thin films as a function of the nonradiative decay rate $k_{N R}$ in the bulk films.

observed in films with the highest $k_{\mathrm{NR}}$ which is dominated by aggregation-induced quenching. The slight deviation of the nearly linear correlation between $\nu$ and the concentration quenching can be explained by variations of the intramolecular non-radiative deactivation rate in different materials. This suggests that the surface quenching and the concentration quenching in the bulk of the film both are controlled by the same mechanism. Previous studies have shown that dipoledipole interactions between $\operatorname{Ir}(\mathrm{ppy})_{3}$ complexes are responsible for concentration quenching in the bulk films. ${ }^{9,12}$ In other words, although triplet exciton diffusion in iridium(III) complex materials is mediated by electron exchange interactions, ${ }^{12}$ the concentration quenching of the phosphorescence in the bulk films and near the dielectric surfaces is controlled by dipole-dipole interactions between iridium(III) complex dendrimer cores.

\subsection{Influence of the refractive index of the substrate}

In order to improve our understanding of the surface quenching processes, we investigated the role of the substrate on the phosphorescence of IrG1 neat films. The PL spectra on different substrates are similar and their peak shifts slightly to shorter wavelengths when decreasing the film thickness (data not shown). Fig. 6 shows the surface quenching dynamics $g(t)$ for IrG1 neat films for different substrates. We find that the effect of the film thickness on the phosphorescence decays varies strongly with the substrate - it is weak in dendrimer films deposited on a sapphire substrate and negligible on SF15 glass. The fits to eqn (3) give values of the surface quenching velocity $v$ which are plotted in Fig. 7 as a function of the refractive index of the substrate at $520 \mathrm{~nm}$ which corresponds to the maximum peak of the IrG1 PL spectrum in the solid state. ${ }^{30}$ Except for the case of ITO and to some extent PEDOT, the surface quenching velocity is found to decrease as the refractive index increases. Note that the influence of the refractive index on $\nu$ cannot be described by a simple $1 / 2 n^{2}$ law ( $n$ being the average refractive index of the medium), which confirms that light-outcoupling does not play any significant role on the investigated quenching processes. In that context, our finding indicates that surface quenching occurs at the film-substrate interface. This behaviour can be due to different densities of trap states at the interface or more likely to the 

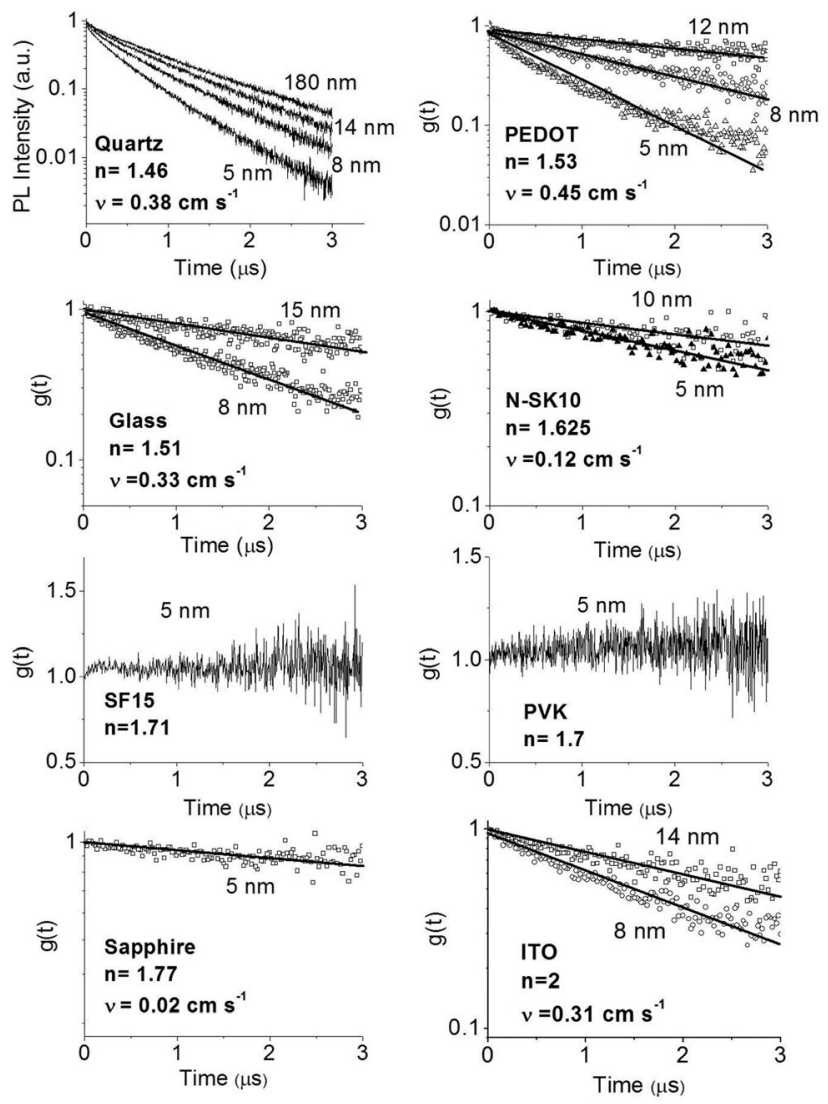

Fig. 6 Ratio of the phosphorescence intensity $g(t)$ measured for neat thin films of IrG1 of given thicknesses to that in the thick film on different substrates, $n$ is the refractive index of the substrate at the PL peak. Solid lines are fits to an exponential decay and surface quenching velocities $v$ obtained using eqn (3) are given.

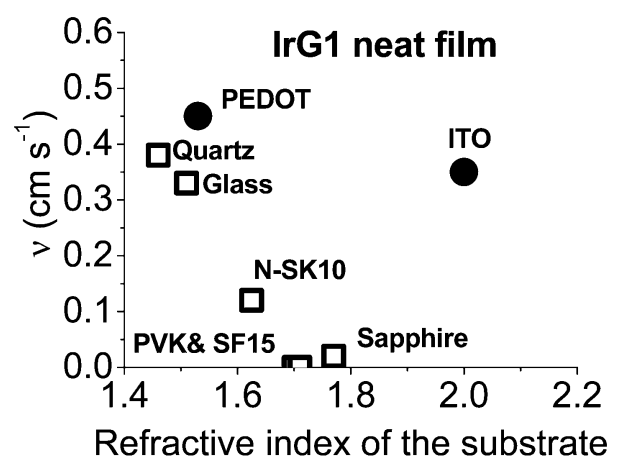

Fig. 7 Surface quenching velocity $v$ for IrG1 neat films as a function of the refractive index of the substrate at $520 \mathrm{~nm}$.

Förster-type energy transfer of the triplet excitons to the substrates, which act as quenchers. ${ }^{9,12}$ The Förster-type energy transfer rate is known to be proportional to $1 / n^{4}$ where $n$ is the refractive index of the medium ${ }^{31,32}$ and the dipole-dipole interactions can be sensitive to the boundary conditions of an interface. ${ }^{33-35}$ Taking this into account, the most plausible explanation for the observed behaviour of the phosphorescence decays would be that the energy transfer of the triplets to the quenchers located at the phosphorescent dendrimer film-substrate interface is controlled by the refractive index of substrate due to a screening of dipole-dipole interactions by the dielectric environment. Note that ITO substrates and PEDOT are commonly employed in practical OLED devices but their use can lead to an additional and alternative pathways to quenching of the excitons at the interface with the organic light-emitting layer. ${ }^{36,37}$ This is consistent with our observation that the surface quenching velocity in both cases is high despite their large refractive indices as compared with those of glass or fused silica. In particular, the strong quenching for ITO suggests that it is directly quenching excitations. This is intriguing in view of its widespread use in efficient OLEDs. However, in efficient OLEDs, the device structure is chosen to ensure that the emission occurs far from the electrodes.

Overall, this study provides new information on the photophysics of solution-processed electrophosphorescent organic solid-state materials. With the current interest in the use of photonic materials to enhance photophysical properties, the possibility to control the energy transfer of triplet excitons to quenching sites by the selection of the substrate may offer new ways of improving light emission from iridium(III) complexes. In addition to OLEDs, the results presented in this work will be essential for the future development of high performance phosphorescent OFETs ${ }^{20}$ where the generation of triplet excitons takes place near the interface with the gate dielectric layer. It is worth noting that the refractive index of the substrate was found to not affect the photophysical properties of fluorescent bisfluorene-cored dendrimers. ${ }^{28}$ The reason why such a different behaviour was observed between phosphorescent iridium(III) complexes and fluorescent dendrimers is still not clear and should be clarified in the near future by examining the photophysical properties of other organic light-emitting materials and determining the nature of the surface quenchers. In that regard, the film thickness dependence of the dynamics of the prompt and delayed fluorescence in TADF materials could be of strong interest.

\section{Conclusion}

In conclusion, we observed a strong thickness dependence of the phosphorescence decays in iridium(III) complex-cored dendrimer films and modelled it by a surface quenching of the excitons at the film interface. The results show that this effect strongly depends on the exciton diffusion and is larger in materials with high non-radiative decay rate and low concentration quenching. Finally, we observed a strong influence of the refractive index of the dielectric substrate on the phosphorescence decays. This is attributed to the role played by the refractive index of the substrate on the Förster type energy transfer rate of triplet excitons to the quenching sites located at the film/substrate interface. This study provides useful new information for the development of high efficiency tripletharvesting electroluminescent devices. 


\section{Acknowledgements}

We are grateful to the EPSRC of the UK for supporting this work through grants EPSRC GR/S82381/01, GR/S82398/01 and $\mathrm{EP} / \mathrm{J} 009016$, and to the European Research Council of the European Union for support from grant number 321305. IDWS is a Royal Society Wolfson Research Merit Award Holder. The research data supporting this publication can be accessed at http:// dx.doi.org/10.17630/30FC4852-3440-4B51-AA95-6A79313D475B.

\section{References}

1 M. A. Baldo, D. F. O'Brien, Y. Tou, A. Shoustikov, S. Sibley, M. E. Thompson and S. R. Forrest, Nature, 1998, 395, 151-154.

2 V. Cleave, G. Yahioglu, P. Le Barny, R. H. Friend and N. Tessler, Adv. Mater., 1999, 11, 285-288.

3 C. Adachi, M. A. Baldo and S. R. Forrest, J. Appl. Phys., 2001, 90, 5048-5051.

4 M. Ikai, S. Tokito, Y. Sakamoto, T. Suzuki and Y. Taga, Appl. Phys. Lett., 2001, 79, 156.

5 X. Yang, D. C. Müller, D. Neher and K. Meerholz, Adv. Mater., 2006, 18, 948-954.

6 K. H. Kim, S. H. Lee, C. K. Moon, S. Y. Kim, Y. S. Park, J. H. Lee, J. W. Lee, J. Huh, Y. M. You and J. J. Kim, Nat. Commun., 2014, 5, 4769.

7 Y. S. Park, S. H. Lee, K. H. Kim, S. Y. Kim, J. H. Lee and J. J. Kim, Adv. Funct. Mater., 2013, 23, 4914-4920.

8 H. Uoyama, K. Goushi, K. Shizu, H. Nomura and C. Adachi, Nature, 2014, 492, 234-238.

9 Y. Kawamura, J. Brooks, J. J. Brown, H. Sasabe and C. Adachi, Phys. Rev. Lett., 2006, 96, 017404.

10 R. J. Holmes, S. R. Forrest, T. Sajoto, A. Tamayo, P. I. Djurovich and M. E. Thompson, Org. Electron., 2006, 7, 163-172.

11 M. A. Baldo, C. Adachi and S. R. Forrest, Phys. Rev. B: Condens. Matter Mater. Phys., 2000, 62, 10967.

12 J. C. Ribierre, A. Ruseckas, K. Knights, S. V. Staton, N. Cumstey, P. L. Burn and I. D. W. Samuel, Phys. Rev. Lett., 2008, 100, 017402.

13 W. I. Jeong, S. Y. Kim, J. J. Kim and J. W. Kang, Chem. Phys., 2009, 355, 25-30.

14 J. C. Ribierre, A. Ruseckas, I. D. W. Samuel, S. Staton and P. L. Burn, Phys. Rev. B: Condens. Matter Mater. Phys., 2008, 77, 085211.

15 J. C. Ribierre, S. G. Stevenson, I. D. W. Samuel, S. V. Staton and P. L. Burn, J. Disp. Technol., 2007, 3, 233-237.
16 J. C. Ribierre, C. Yates, A. Ruseckas, S. V. Staton, P. L. Burn and I. D. W. Samuel, Org. Electron., 2010, 11, 62-66.

17 J. P. J. Markham, I. D. W. Samuel, S.-C. Lo, P. L. Burn, M. Weiter and H. Bässler, J. Appl. Phys., 2004, 95, 438-445.

18 E. B. Namdas, A. Ruseckas, I. D. W. Samuel, S.-C. Lo and P. L. Burn, Appl. Phys. Lett., 2005, 86, 091104.

19 E. B. Namdas, A. Ruseckas, I. D. W. Samuel, S.-C. Lo and P. L. Burn, J. Phys. Chem. B, 2004, 108, 1570-1577.

20 E. B. Namdas, B. B. Y. Hsu, Z. Liu, S.-C. Lo, P. L. Burn and I. D. W. Samuel, Adv. Mater., 2009, 21, 4957-4961.

21 D. F. O'Brien, C. Giebler, R. B. Fletcher, A. J. Cadby, L. C. Palitis, D. G. Lidzey, P. A. Lane, D. D. C. Bradley and W. Blau, Synth. Met., 2001, 116, 379-383.

22 N. C. Greenham, I. D. W. Samuel, G. R. Hayes, R. T. Phillips, R. R. Kessener, S. C. Moratti, A. B. Holmes and R. H. Friend, Chem. Phys. Lett., 1995, 241, 89-96.

23 K. H. Tews, O. Inacker and H. Kuhn, Nature, 1970, 228, 278-280.

24 K. H. Drexhage, Prog. Opt., 1974, 12, 165-231.

25 W. L. Barnes, J. Mod. Opt., 1998, 45, 661-699.

26 G. L. J. A. Rikken, Phys. Rev. A: At., Mol., Opt. Phys., 1995, 51, 4906. 27 S. V. Vickers, H. Barcena, K. A. Knights, R. K. Thomas, J. C. Ribierre, S. Gambino, I. D. W. Samuel, P. L. Burn and G. Fragneto, Appl. Phys. Lett., 2010, 96, 263302.

28 J. C. Ribierre, A. Ruseckas, P. E. Shaw, H. S. Barcena, P. L. Burn and I. D. W. Samuel, J. Phys. Chem. C, 2008, 112, 20463-20468.

29 J. C. Ribierre, A. Ruseckas, I. D. W. Samuel, H. S. Barcena and P. L. Burn, J. Chem. Phys., 2008, 128, 204703.

30 S.-C. Lo, N. A. H. Male, J. P. J. Markham, S. W. Magennis, P. L. Burn, O. V. Salata and I. D. W. Samuel, Adv. Mater., 2002, 14, 975-979.

31 T. Förster, Ann. Phys., 1948, 2, 55-75.

32 R. S. Knox and H. van Amerongen, J. Phys. Chem. B, 2002, 106, 5289-5293.

33 P. Andrew and W. L. Barnes, Science, 2000, 290, 785-788.

34 M. Hopmeier, W. Guss, M. Deussen, E. O. Göbel and R. F. Mahrt, Phys. Rev. Lett., 1999, 82, 4118-4121.

35 G. S. Agarwal and S. D. Gupta, Phys. Rev. A: At., Mol., Opt. Phys., 1998, 57, 667.

36 J. Mei, M. S. Bradley and V. Bulovic, Phys. Rev. B: Condens. Matter Mater. Phys., 2009, 79, 235205.

37 K. H. Yim, R. H. Friend and J. S. Kim, J. Chem. Phys., 2006, 124, 184706. 